\title{
Validation of an integrated patient positioning system: Exactrac and iViewGT on Synergy Platform
}

\author{
Kanan Jassal 1, 2*, Anusheel Munshi 1, Biplab Sarkar 1, Sayan Paul 1, Aman Sharma 1, \\ Bidhu Kalyan Mohanti 1, T Ganesh 1, Arun Chougule 2, Kanupriya Sachdev ${ }^{3}$ \\ ${ }^{I}$ Department of Radiation Oncology, Fortis Memorial Research Institute, Gurgaon, India. \\ ${ }^{2}$ Department of Radiological Physics, SMS Medical College and Hospital, Jaipur, India. \\ ${ }^{3}$ Department of Physics, Malviya National Institute of Technology, Jaipur, India.
}

Received February 25, 2014; Revised March 31, 2014; Accepted April 01, 2014; Published Online April 09, 2014

\section{Original Article}

\begin{abstract}
Purpose: Evaluation of the newly integrated system for its validation and designing a quality assurance frame work to assess its geometrical, radiological and mechanical accuracy. Methods: Isocentric accuracy of two independent imaging modalities, $\mathrm{kV}$ based ExacTrac and MV based iViewGT was evaluated using Winston-Lutz test. A pelvic humanoid phantom was used for the radiological end-to-end test for its clinical utilization. Image quality for the systems was evaluated using Las Vegas Phantom and ETR-1 plate. The $\mathrm{kV}$ system was also assessed for $\mathrm{kVp}$ accuracy, $\mathrm{kVp}$ - dose linearity, $\mathrm{mAs}$-dose linearity and timer linearity and its accuracy. The system was tested for total filtration and output consistency. Tests for uniformity and noise measurement of $\mathrm{kVp}$ accuracy and its reproducibility, linearity test between applied $\mathrm{kVp}$ and the $\mathrm{x}$-ray dose, linearity Test between applied $\mathrm{mAs}$ and the $\mathrm{x}$-ray dose were also done. Results: Winston-Lutz test gave the isocentric deviation of $0.058 \pm 0.015 \mathrm{~mm}$ with the average lateral deviation as $0.028 \pm 0.021 \mathrm{~mm}$, average longitudinal deviation as $0.032 \pm 0.015 \mathrm{~mm}$ and average vertical deviation as $0.030 \pm 0.016 \mathrm{~mm}$. With the phantom test, the minimum measured displacement of Exactrac positioning was $0.2 \pm 0.3 \mathrm{~mm}, 0.0 \pm$ $0.2 \mathrm{~mm}$ and $0.1 \pm 0.3 \mathrm{~mm}$ in longitudinal, lateral and vertical directions respectively. In image quality test, visible smallest visible hole size seen by both Exactrac and EPID imaging system was $5 \mathrm{~mm}$ and can resolve $1.5 \mathrm{lp} / \mathrm{mm}$ or better. The image uniformity was found to be $132.9 \pm 3.06$ pixels for MV images and $139 \pm 4.41$ pixels for $\mathrm{kV}$ images with the associated noise of $\leq 1 \%$ both for $120 \mathrm{kV}-20 \mathrm{mAs}$ and $4 \mathrm{MV}$ beam energy of ExacTrac and iViewGT respectively. The uniformity and noise test, measured pixel intensity values for various points on $\mathrm{MV}$ and $\mathrm{kV}$ images separately were found to agree within $\pm 1 \%$ with respect to the central axis pixel value. The $\mathrm{kVp}$ accuracy and its reproducibility were tested for $\mathrm{kV}$ imager only. The deviation of $\mathrm{kVp}$ was found to be than $\pm 1 \%$ and its precision was seen to be even lesser than $\pm 0.1 \%$. Linearity test between applied $\mathrm{kVp}$ and the $\mathrm{x}$-ray dose and applied $\mathrm{mAs}$ and $\mathrm{x}$-ray dose were tested only for the ExacTrac. Both the coefficient of linearity for $\mathrm{kVp}$ as well as $\mathrm{mAs}$ was found to be $<0.1$. Conclusion: It is feasible to install ExacTrac imaging system with an Elekta linear accelerator. Both the imaging systems were found to be compatible in terms of image quality test and isocentric accuracy and can be used for the patient imaging in the same Linear accelerator.
\end{abstract}

Keywords: Image Guided Radiotherapy; Patient Set-Up; Exactrac; Quality Assurance

\section{Introduction}

Patient set-up during radio-therapeutic procedures is a critical concern as it has its prime impact on tumor control.

Corresponding author: Kanan Jassal; Department of Radiation Oncology, Fortis memorial research institute, Gurgaon, India.

Cite this article as:

Jassal K, Munshi A, Sarkar B, Paul S, Sharma A, Mohanti BK, Ganesh T, Chougule A, Sachdev K. Validation of an integrated patient positioning system: Exactrac and iViewGT on Synergy Platform. Int J Cancer Ther Oncol 2014; 2(2):020212.

DOI: $10.14319 /$ ijcto.0202.12

A part of this work was presented at $34^{\text {th }}$ meeting of Association of Medical Physicists of India (AMPI) "International Conference on Medical Physics-2013" at Saha Institute of Nuclear Physics, Kolkata in the best paper session in November 13-15, 2013.
Minor deviations in delivering radiation beams from the actual simulated position can not only deteriorate tumor control but also have a detrimental impact on surrounding normal tissues. The severity of normal tissue complications depends on the spatial anatomy, irradiated tissue volume and the delivered dose. Therefore, it is crucial to accurately reproduce the patient setup as per the simulation during treatment planning. Given this, recent research has witnessed considerable engagement in developing treatment machines, procedures and protocols for patient set-up and position verification. 
Such position verification include image guided treatment delivery systems further classified as linear accelerator embedded imaging like EPID, CBCT, independent stereoscopic imaging systems like ExacTrac, ultrasound based imaging ${ }^{1,2}$ and infrared body marker tracking. ${ }^{3,4}$ Among these, portal-imaging technique is notably the most robust system due to its inherent geometrical advantage that has been tested clinically by several investigators over the years. However, it has been subjected to a debate between geometrical accuracy and image quality as it suffers from compromised image quality due to higher energy used than kilo voltage $(\mathrm{kV})$ that yields images of better quality. As a consequence, a linear accelerator integrated with in-room imaging technology that uses $\mathrm{kV}$ x-ray sources with flat panel imager has emerged as an integrated image guided radiation therapy (IGRT) system for patient positioning and verification. One of this kind of integration is done at our institute using Exactrac stereoscopic $\mathrm{kV} X$ ray Imaging and Synergy Platform (Elekta AB, Stockholm, Sweden) linear accelerator equipped with iVeiwGT (Elekta, Crowley, UK) a-Si Flat mega voltage (MV) imaging panel. Although the Exactrac system is well established with the Varian (Varian Medical System, Polo Alto, CA) linear accelerator ${ }^{5}$, the new conjugation with Elekta Medical linear accelerator was evaluated rigorously. The current study illustrates the commissioning, installation and acceptance of the hybrid system along with the designing of a comprehensive quality assurance (QA) framework program to assess its mechanical, geometrical, and radiological accuracy.

\section{Methods and Materials}

\section{Medical linear accelerator Elekta Synergy platform with BrainLAB ExacTrac as an integrated patient posi- tioning system:}

Elekta Synergy Platform (Elekta AB, Stockholm, Sweden) is the state-of-the art digital linear accelerator with the capabilities of step and shoot IMRT, dynamic IMRT and VMAT (volumetric modulated arc radiotherapy). It is also equipped with the MV electronic portal-imaging device (EPID).

\section{MV amorphous Silicon electronic portal imaging de- vice (a-Si EPID)}

Amorphous silicon electronic portal imaging device (a-Si EPID) consists of a detector panel from Perkin- Elmer (Fremont, CA, USA) that is mounted on the linear accelerator. The active matrix has $41 \times 41 \mathrm{~cm}^{2}$ dimension consisting of a copper plate and a $133 \mathrm{mg} / \mathrm{cm}^{-2}$ terbium-doped gadoliniumoxysulphide $\left(\mathrm{Gd}_{2} \mathrm{O}_{2} \mathrm{~S}\right.$ : $\left.\mathrm{Tb}\right)$ screen with the maximum imaging area of $25.6 \times 25.6 \mathrm{~cm}^{2}$ at its isocenter. Source to the detector distance (SDD) is $160 \mathrm{cms}$ with the resultant image of $1024 \times 1024$ pixels. Based on the specifications given by Elekta the resolution is 2.0 pixels $/ \mathrm{mm}$ and Nyquist frequency $\left(\mathrm{f}_{\mathrm{n}}\right)$ is $1 \mathrm{lp} / \mathrm{mm} \cdot{ }^{6,7}$

\section{BrainLAB ExacTrac System}

The ExacTrac patient positioning system from BrainLAB has two modules: Infra-red and dual diagnostic $\mathrm{kV}$-ray tubes with a-Si panel detectors. The first module consists of two infra-red cameras, one video camera and a set of reflective infra-red markers that acts as a remote IR guided couch controller. The second module consists of two floor embedded $\mathrm{x}$-ray tubes (Varian Medical Systems, Palo Alto, CA) projecting towards the respective ceiling which is mounted with fixed a-Si flat panel detectors (Model XRD 512- 400 AL1: Perkin-Elmer, Waltham, MA) for the purpose of image detection. The flat-panels have a $0.4 \mathrm{~mm}$ as a pixel size and the maximum area that can be imaged is $20.5 \times 20.5 \mathrm{~cm}^{2}$ with the resultant image with $512 \times 512$ pixels.

\section{Geometric and radiological co-incidence of MV iso- center to $\mathrm{kV}$ isocenter}

The isocenter was validated geometrically using a combination of pin-pointer fixed on couch-end and front pointer fixed on collimator. Gantry, collimator head and treatment couch were adjusted and corrected for all types of mechanical instabilities. Subsequently, the co-incidence of the rotation axes of gantry, couch and collimator were verified using radiological spoke film tests. The ExacTrac system was adjusted mechanically with respect to the consistent isocenter position located using calibrated room lasers. It is important to note that the geometric correlation between linear accelerator and ExacTrac system are based on the accuracy of lasers.

A simple way to check the isocenter co-incidence of this hybrid system is to test images of the Winston-Lutz pointer (W-L pointer) from both imaging modalities. This pointer consists of a $5 \mathrm{~cm}$ cube of tissue-equivalent material with a 2 $\mathrm{mm}$ steel ball embedded in the center. For our purpose, the $\mathrm{W}-\mathrm{L}$ pointer was positioned close to the gantry isocenter as aligned using lasers. The study focuses on a set of measurements using Exactrac to obtain systematic error relative to the radiological isocenter. The relative shift(s) of $\mathrm{W}-\mathrm{L}$ pointer with respect to the Exactrac isocenter can be denoted as the following:

\footnotetext{
${ }^{\text {WLVEREXACT }}=$ Vertical shift of WL pointer w.r.t. ExacTrac isocenter

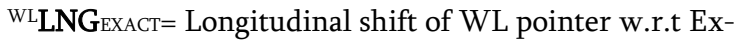
acTrac isocenter

${ }^{\mathrm{WL} L A T E X A C T=}$ Lateral shift of WL pointer w.r.t ExacTrac isocenter
}

Keeping the pointer in the similar position, its EPID images were acquired at various paired gantry angles viz. $0^{\circ}-90^{\circ}$ and $180^{\circ}-270^{\circ}$. The obtained images were matched with its reference images using the iViewGT software for every pair, then mean shift was calculated. Following this, as described 
below, similar shifts with respect to the EPID isocenter were also noted.

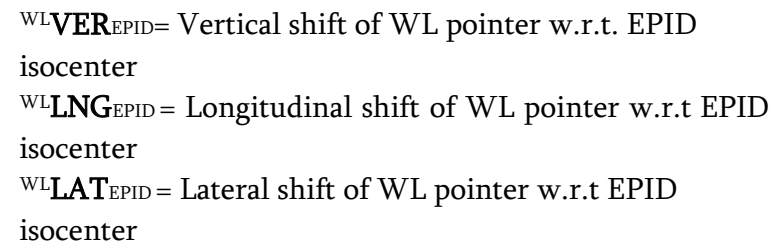

Finally, the deviation measured from both EPID and ExacTrac were evaluated as deviation from radiological co-incidence.

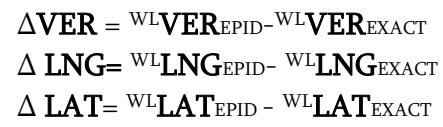

These deviation parameters contribute in validating the accuracy for the shifts obtained individually by both the imaging modalities such that the shifts must lie within the tolerance limits for the isocenter.

\section{Phantom positional shift test}

To test the overall congruence and functionality of the system, a pelvic phantom with an implanted marker of $2 \mathrm{~mm}$ diameter, considered as a target, was used. The phantom was scanned by PET/CT Truflight TOF (Philips Medical Systems Cleveland, Ohio) as a simulator, for a slice thickness of 2 $\mathrm{mm}$. The set of CT images were imported to the treatment planning system CMS Xio version 4.70 .00 (Elekta Inc., Norcross, GA). A simple four-field plan (at $0^{\circ}, 90^{\circ}, 180^{\circ}$ and $270^{\circ}$ gantry angle) was generated for the implanted marker's center as the isocenter for the incident beams. The relevant information was transferred to the respective control - console of the units and the phantom was measured in the same way as the $\mathrm{W}$-L pointer. Keeping the computations same for the phantom, results from both the tests were compared to evaluate consistency of the shifts.

\section{Image quality evaluation tests}

\section{Tests for spatial resolution and contrast}

Image quality tests were performed on the EPID and ExacTrac images separately and independently. Images captured by the hybrid system are the EPID images that does not discriminate soft tissues, while the $\mathrm{kV}$ ExacTrac images provide information of low contrast structures. The overall system has a capacity of doing registration based on high as well as low contrast. The line pair template made of $0.1 \mathrm{~mm}$ thick lead on the ETR-1Test Tool (iba Dosimetry, GmBH), is a multipurpose test tool. For the spatial resolution measurements, a template (shown in Figure 1(a)) was used for ExacTrac. The test pattern had 20 line pairs in the range of 0.6 $\mathrm{lp} / \mathrm{mm}$ to $5.0 \mathrm{lp} / \mathrm{mm}$. The common practice to describe the spatial frequency is the line width and separation distance in terms of line pair (lp) per unit distance (in millimeters). To evaluate an imaging system, the visible line pair is recorded and as per AAPM, $1981{ }^{8}$ and RSD, AERB ${ }^{9}$ below $0.8 \mathrm{lp} / \mathrm{mm}$ is not the acceptable. For determining the contrast and resolution for EPID, the study used Las Vegas Phantom that was placed at the isocenter to acquire planar MV images using 1-2 monitor units (MU) at lowest photon energy available (4MV). The Las Vegas Phantom (shown in Figure 1) is an aluminum slab with 28 circular holes of different depths and diameters engraved in a matrix of 6 columns and 5 rows. An EPID image of this phantom was used to indicate the ability of the imaging system to resolve small and low contrast features. The test was repeated for the other photon energies as well.

\section{Test for uniformity and noise}

Image quality expressed in physical parameters like uniformity and noise were also assessed in the study. For this purpose, MV EPID images in a pair of anterio-posterior (AP) and right lateral (RT LAT) and stereoscopic $\mathrm{kV}$ ExacTrac images were obtained by stacking up to $5 \mathrm{~cm}$ the $30 \times 30 \times 1$ $\mathrm{cm}^{3}$ slabs of virtual water phantom (PTW, Freiburg, Germany) on the carbon fiber couch top. Then, to observe the uniformity in these set of images, 5 points were selected: one on the central axis, four other points were selected in off-axis directions vis-à-vis right, left, and towards gun and towards target directions. The pixel intensity values of these points were observed in a java based image-processing based software named Image J version 1.46r (National Institute of Health, USA). For quantifying image noise above the pixel values, mean pixel value and the standard deviation of the recorded data was estimated.

\section{Tests related to determine the characteristics of $\mathrm{kV}$ $\mathbf{x}$-ray tubes for ExacTrac}

We also examined certain parameters that affect the X-ray quality, by investigating the relationship between the exposure/dose/output of the units and the parameters that include $\mathrm{mAs}$ and $\mathrm{kVp}$. Following section of the paper describe the tests performed for both the ExacTrac units independently. The tests are namely -

- Test for accuracy and reproducibility for $\mathrm{kVp}$

- Linearity test between applied $\mathrm{kVp}$ and the $\mathrm{x}$-ray dose

- $\quad$ Linearity test between applied $\mathrm{mAs}$ and the $\mathrm{x}$-ray dose

- Linearity and accuracy test for timer

- Total filtration evaluation by HVL (Half Value Layer) determination method

- Output consistency test 

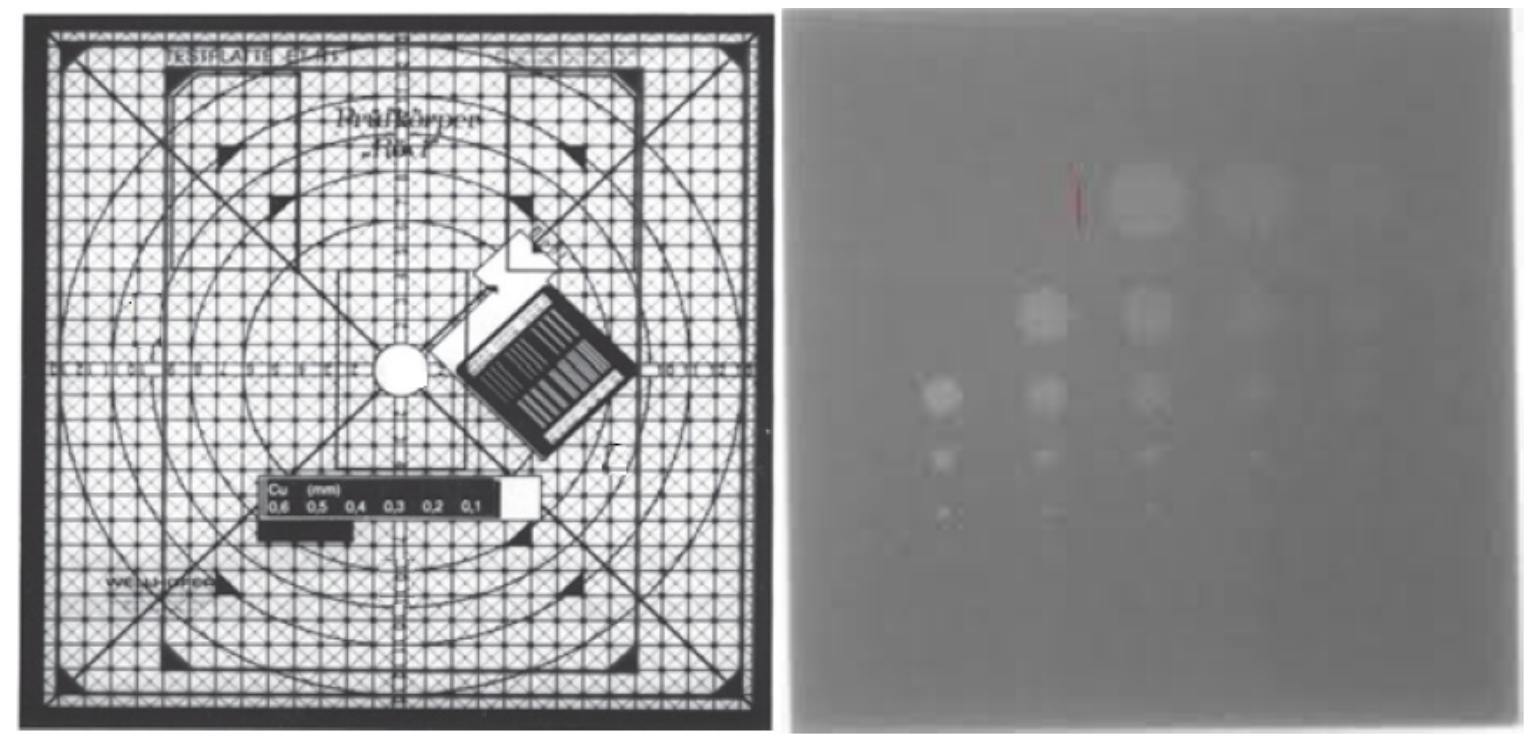

FIG.1: Radiological images of (left) Test plate ETR-1, and (right) Las Vegas phantom.

\section{Measurement of $\mathrm{kVp}$ accuracy and its reproducibility}

For the $\mathrm{X}$-ray tubes, $\mathrm{kVp}$ values were determined by the MagicMaX and multi detector (iba dosimetry, $\mathrm{GmBH}$ ) by applying mAs settings as a constant. The measured values of the $\mathrm{kVp}$ were plotted against the values applied $\mathrm{kVp}$ on the $\mathrm{x}$-ray units.

\section{Linearity test between applied $\mathrm{kVp}$ and the $\mathrm{x}$-ray dose}

The dosimeter MagicMaX and multi detector (iba dosimetry, $\mathrm{GmBH}$ ) was positioned at central axis of the projected beam such that the $\mathrm{X}$-ray tube focal spot-to-detector distance (FDD) was $100 \mathrm{~cm}$ for the measurements and the detector was placed on the treatment couch of LINAC. In order to investigate the effect of $\mathrm{kVp}$ to the dose, the $\mathrm{x}$-ray units were set at $10 \mathrm{mAs}$ and with an incremental value of $\mathrm{kVp}$ with a range of 50 to $150 \mathrm{kVp}$. X-ray exposures were made and the dosimeter readings were recorded by repeating different $\mathrm{kVp}$ settings $(50,70,80100,120$ and $150 \mathrm{kVp})$ at constant mAs. The measured dose values were plotted against the corresponding $\mathrm{kVp}$ for both $\mathrm{X}$-ray units separately.

\section{Linearity test between applied $\mathrm{mAs}$ and the $\mathrm{x}$-ray dose}

In order to determine the effect of $m A s$ on dose, the exposures were performed with constant $\mathrm{kVp}(60 \mathrm{kVp})$, but with gradually increasing $\mathrm{mAs}(8,10,12.5,20,40$ and $50 \mathrm{mAs})$. Similar X-ray dose measurements were also determined at 100 and $140 \mathrm{kVp}$ settings for each $\mathrm{mAs}$ value $(8,10,12.5,20$, 40 and $50 \mathrm{mAs}$ ). The measurement results for each X-ray unit were plotted against the corresponding $\mathrm{mAs}$.

\section{Linearity and accuracy test for timer}

To evaluate the accuracy and linearity of timer for exposure the multi-detector dosimeter was positioned at focus-to-detector-distance (FDD) of $100 \mathrm{~cm}$. For the constant
$\mathrm{mAs}$ value, the exposure time was recorded using the detector associated software. Thus, the error between the set time and the observed time was calculated to determine the accuracy of the timer.

In order to determine the linearity of timer, the exposures were performed with constant values of $\mathrm{kVp}$ ranging between $50 \mathrm{kVp}$ to $120 \mathrm{kVp}$, but with gradually increasing mAs values $(8,10,12.5,20,40$ and $50 \mathrm{mAs})$. The dosimeter associated MagicMaX software was used to determine the timer values during the exposures.

\section{Total filtration evaluation}

For the HVL measurements, filtration was realized by using aluminum (Al) filters with the incremental thickness of 1 $\mathrm{mm}$. During the measurements, $\mathrm{mAs}$ and $\mathrm{kVp}$ were stable $(20 \mathrm{mAs}, 100 \mathrm{kVp}$ ) and the distance was determined as $100 \mathrm{~cm}$. Initially, the dose measurement without the filter was generalized and further the dose measurement was repeated using filter of varying thickness. Each filter thickness was obtained by adding $1 \mathrm{~mm}$ of $\mathrm{Al}$. The dose measurements were taken for varying conditions - without filter, and with filters starting from $1 \mathrm{~mm}$ of $\mathrm{Al}$ up to $20 \mathrm{~mm}$ of $\mathrm{Al}$.

\section{Output consistency test}

A MagicMaX and multi detector assembly (iba dosimetry, $\mathrm{GmBH}$ ) was used to evaluate does to the patient at isocenter by exposure measurements for individual $x$-ray units. The chamber was placed on the treatment couch. The operating potential was varied from 40 to $150 \mathrm{kVp}$ and resultantly operating current for the set voltage varied from $63 \mathrm{~mA}$ to 200 $\mathrm{mA}$. Also, the exposure time varied from $100 \mathrm{mSec}$ to 30 $\mathrm{mSec}$ for each type of $\mathrm{kVp}-\mathrm{mA}$ combinations. The chamber measured charge collected at each exposure in the range of nano and pico-coulomb, and software directly calculated the 
output in micro-Gray ( $\mu \mathrm{Gy})$, using the calibration data provided by the secondary standard accredited laboratory and is traceable to the primary standard laboratory.

\section{Results}

The detailed and comprehensive evaluation was performed for medical linear accelerator and the add-on imaging system of BrainLAB. This study presents the results of initial acceptance testing, commissioning and validation of the complete system. Also, this data forms the baseline for any future evaluation of measurements and functionality of the integrated system. The following sub-section of the paper discusses the results of various tests performed.

\section{Test for the mechanical integrity of the system}

The safety features of the EPID system were tested over ten days. An average of $55.22 \pm 0.057 \mathrm{~cm}$ was found to be the distance measured between EPID panel and isocenter at various gantry positions $\left(0^{\circ}, 90^{\circ}, 180^{\circ}, 270^{\circ}\right)$. Also the ODI readings were measured along the central axis. In the anterior and posterior directions, the distance was measured within \pm $1 \mathrm{~mm}$ from the manufacturer's specifications that was taken as the reference values. Similarly in the lateral directions, the distance was measured within $\pm 0.5 \mathrm{~mm}$ from the reference values.

\section{Geometric and radiological co-incidence of MV iso- center to $\mathrm{kV}$ isocenter}

The images that were obtained of WL phantom ensured the coincidence with the isocenter of linear accelerator and ExacTrac. The test was repeated for the 10 days and the resultant deviation from radiological co-incidence (shown in Figure 2) was calculated as $0.058 \pm 0.015 \mathrm{~mm}$ with the average lateral deviation as $0.028 \pm 0.021 \mathrm{~mm}$, average longitudinal deviation as $0.032 \pm 0.015 \mathrm{~mm}$ and average vertical deviation as $0.030 \pm 0.016 \mathrm{~mm}$.

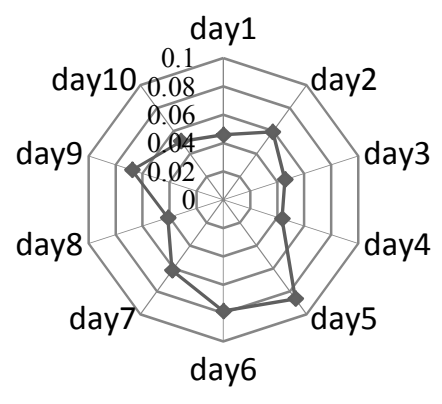

FIG. 2: Deviation of WL pointer from its radiological co-incidence.

\section{Phantom positional shift test}

The pelvic part of the Rando phantom was used for the verification for the isocenter that marked the end-to-end testing of the system. The shifting co-ordinates were obtained from the CMS Xio version 4.70 .00 treatment planning system. Subsequently, it was imaged using both the independent imaging systems. Certain intentional shifts up to $2 \mathrm{~cm}$ were given to phantom. The EPID and Exactrac images were taken for the phantom in the known displacement position. Phantom repositioning were made according to the Exactrac positioning system and was verified using EPID. The minimum measured displacement of Exactrac positioning was 0.2 $\pm 0.1 \mathrm{~mm}, 0.0 \pm 0.02 \mathrm{~mm}$ and $0.1 \pm 0.05 \mathrm{~mm}$ in longitudinal, lateral and vertical directions respectively. Maximum measured discrepancies of the re-positioning of the target during intentional shifts were recorded within $0.2 \pm 0.04 \mathrm{~mm}$.

\section{Image quality evaluation tests}

\section{Tests for spatial resolution and contrast}

Image quality of a newly integrated system was tested for the spatial and contrast resolution of the flat panels. For EPID, these tests were performed using Las Vegas phantom. As per the thumb-rule for contrast, the maximum $\mathrm{R}$ (row) greater than R2 (row 2) in the maximum C (column) tolerably greater than C5 (column 5) was visible for all photon energies. Similarly, for spatial resolution, the maximum C (column) greater than C3 (column 3) in the maximum R (row) tolerably greater than C3 (column 3) was visible for all photon energies. For ExacTrac, the tests were performed using ETR-1 test plate. The images were acquired with the parameter settings at $40 \mathrm{kVp}$, with $40 \mathrm{mAs}$ for both the floor embedded x-ray units. High and low contrast sensitivity for both the panels were found to be within the recommended performance standard as per regulatory authority in India. ${ }^{9}$ The diameter of the smallest size hole clearly visible on both the imaging panels was noted to be $5 \mathrm{~mm}$ and the visible bar pattern of $1.5 \mathrm{lp} / \mathrm{mm}$ or better was resolved.

\section{Test for uniformity and noise}

The measured pixel intensity values for various points on $\mathrm{MV}$ and $\mathrm{kV}$ images separately were found to agree within \pm $1 \%$ with respect to the central axis pixel value. Statistically the mean pixel value and standard deviation values were evaluated as $132.9 \pm 3.06$ for MV images and $139 \pm 4.41$ for $\mathrm{kV}$ images.

\section{Tests related to determine the characteristics of $\mathrm{kV}$ $\mathrm{x}$-ray tubes for ExacTrac}

\section{Measurement of $\mathrm{kVp}$ accuracy and its reproducibility} The accuracy of $k \mathrm{Vp}$ was determined with respect to the applied $\mathrm{kVp}$ values. Plotted graph between applied and measured $\mathrm{kVp}$ has been shown in Figure 3(a) for both of the $\mathrm{x}$-ray units. The deviation was found to be lesser than $\pm 1 \%$ and its precision was seen to be even lesser than $\pm 0.1 \%$. 


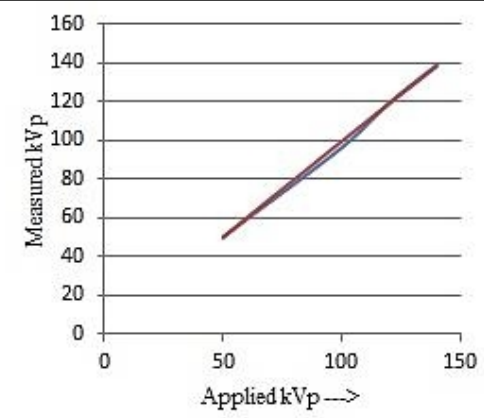

(a) Measurementof klp accwacy \& its reproducibility

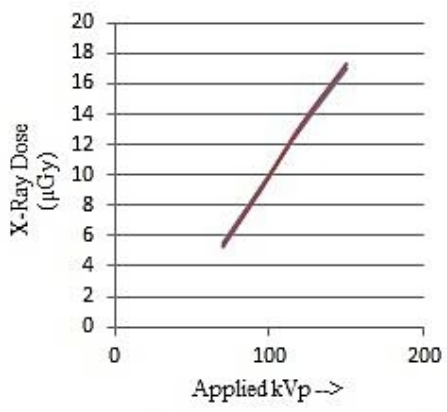

(b) Linearity Test for applied kLp \& the x-ray dose

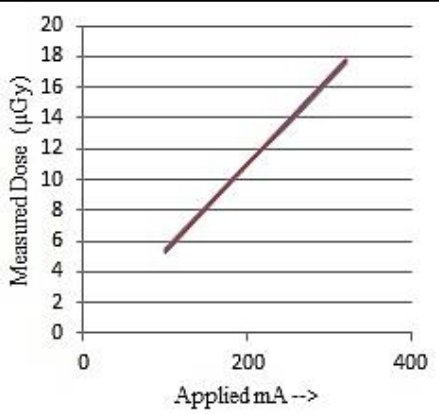

(c) Linearity Test for applied mAs \& $x$-ray dose

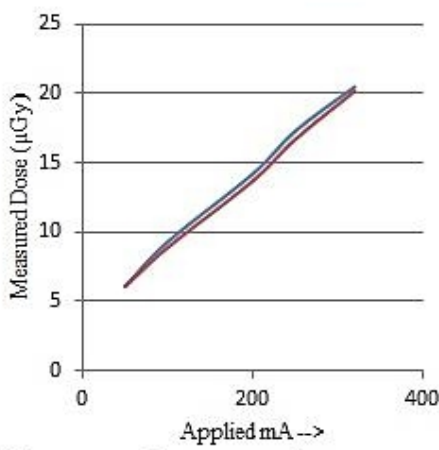

(d) Linearity and accuracy test for timer

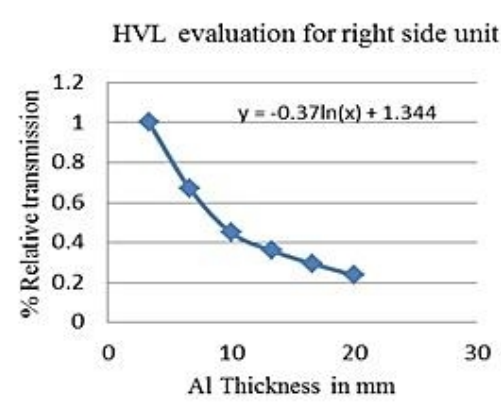

(e)i. Total filtration \&HVL test for right unit

HVL evaluation for left side unit

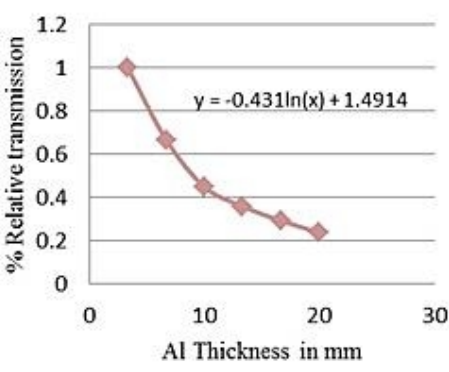

(e) ii. Total filtration \& HVL test for left unit

FIG. 3: Related test to determine the characteristics of $\mathrm{kV} x$-ray tubes for ExacTrac

Linearity Test between applied $\mathrm{kVp}$ and the $\mathrm{x}$-ray dose

The relationship between $\mathrm{x}$-ray dose and $\mathrm{kVp}$ was found to be linear. The coefficient of linearity (COL) is defined as where,

$$
\text { Coefficient of Linearity }=\frac{\operatorname{Dmax}-\text { Dmin }}{\text { Dmax }+ \text { Dmin }}
$$

$D_{\max }$ is the maximum value of Dose and;

$D_{\min }$ is the minimum value of Dose

The significance of this parameter is that it gives us a measure of the reliability of the linear relationship between the dose and $\mathrm{kVp}$ values. The value of COL was evaluated for both the ExacTrac X-ray units and found as $3.05 \times 10^{-4}$ for right side $\mathrm{x}$-ray tube and $3.28 \times 10^{-4}$ for left side $\mathrm{x}$-ray tube. These values are much lesser than the maximum tolerance limit $(\mathrm{COL}<0.1)$ of this parameter. Plotted graph between applied $\mathrm{kVp}$ and measured dose has been shown in Figure 3 (b) for both of the $x$-ray units.

\section{Linearity Test between applied $\mathrm{mAs}$ and the $\mathrm{x}$-ray dose}

The relationship between $\mathrm{x}$-ray dose and $\mathrm{mA}$ was found to be linear. Plotted graph between applied $\mathrm{mAs}$ and measured dose has been shown in Figure 3(c) for both of the x-ray units. Also statistically, the coefficient of linearity (COL) was determined and was found to be in range of $9.44 \times 10^{-4}$ for right side $\mathrm{x}$-ray tube and $9.51 \times 10^{-4}$ for left side $\mathrm{x}$-ray tube.
This value is much less than the maximum tolerance limit value of COL parameter $(\mathrm{COL}<0.1)$.

\section{Linearity and accuracy test for timer}

The timer was found to have a good accuracy and reproducibility for both the x-ray units (shown in Figure 3(d)). The accuracy of the timer was evaluated with respect to the set value in the ExacTrac software. The error was evaluated to be lesser than $\pm 1 \%$. Similarly, reproducibility was evaluated even lesser than $\pm 0.3 \%$. It was found that for the constant $\mathrm{kVp}$ values against variable $\mathrm{mA}$ values, the measured dose was found to be linear for both our units.

\section{Total filtration and HVL evaluation tests}

The dose measurement results were plotted against the aluminum (Al) thickness. Dose (mGy) calculations were derived from the experimental data values as a function of Al thickness. And subsequently, the $\mathrm{Al}$ thickness in which the dose decreased to its half value was calculated. The final values of filtration of the $\mathrm{x}$-ray tubes were evaluated from the standard curves available for different applied potential to the three phase generator X-ray units. These standard curves are plotted between the measured HVL value ( $\mathrm{mm}$ of $\mathrm{Al}$ ) and the total filtration value ( $\mathrm{mm}$ of $\mathrm{Al}$ ) as shown in Figure 3 (e(i)) and 3 (e(ii)). The inherent filtration for right side of $\mathrm{x}$-ray tube was calculated as $2.5 \mathrm{~mm}$ thickness of $\mathrm{Al}$ and for left side of x-ray tube was $2.3 \mathrm{~mm}$ thickness of Al. 
TABLE 1: The consistency of the output from the $\mathrm{x}$-ray tubes and applied $\mathrm{kVp}$.

\begin{tabular}{c|c|cc|cc}
\hline \hline & & \multicolumn{2}{|c|}{ Right Side X-ray tube } & \multicolumn{2}{c}{ Left Side X-ray tube } \\
\cline { 3 - 6 } S. No. & Applied kVp & $\begin{array}{c}\text { Mean output } \\
(\mu \mathrm{G})\end{array}$ & $\begin{array}{c}\text { Standard } \\
\text { deviation }\end{array}$ & $\begin{array}{c}\text { Mean output } \\
(\mu \mathrm{G})\end{array}$ & $\begin{array}{c}\text { Standard } \\
\text { deviation }\end{array}$ \\
\hline 1 & 70 & 5.53 & 0.12 & 7.61 & 0.11 \\
2 & 80 & 6.72 & 0.18 & 9.68 & 0.23 \\
3 & 100 & 11.46 & 0.07 & 15.66 & 0.20 \\
4 & 120 & 14.46 & 0.08 & 17.53 & 0.05 \\
\hline \hline
\end{tabular}

\section{Output consistency test}

In order to investigate the consistency of the output from the $\mathrm{x}$-ray tubes and $\mathrm{kVp}$, firstly the $\mathrm{x}$-ray tube outputs for 10 $\mathrm{x}$-ray units, were calculated by dividing the measured dose values to the $\mathrm{mAs}$ values. Table 1 provides our results for the output consistency of both the $\mathrm{x}$-ray tubes.

\section{Discussion}

The focus of this work is to access the recent integration between BrainLAB Exactrac patients positioning system (ExacTrac; BrainLAB, Heimstetten, Germany) with Elekta's Synergy platform (Elekta AB, Stockholm, Sweden) at Fortis Memorial Research Institute (FMRI), Gurgaon. The functionality of this hybrid system depend on the various aspects such as for $\mathrm{kV}$ imaging, relies upon mechanical integrity and stability of the two X-ray tubes, flat panel imagers and fidelity of the operating software. In the validation program, we evaluated EPID of Synergy Platform as IGRT system and the characteristics of $\mathrm{kV}$ X-ray units of ExacTrac device for patient positioning. Mechanical front pointer is a traditional tool for isocenter verification. The method is cumbersome and the result also varies due to human observation. Additionally, mechanical front pointers are also susceptible to physical damages and cannot be considered as reliable device. Similarly, wall lasers are also prone to mechanical instabilities on daily basis. The Winston - Lutz phantom was thus, selected as a radiological test for the hidden target. This technique was introduced by Lutz, Winston and Maleki at Harvard Medical School in 1988. ${ }^{10}$ The technique is very common and simple to perform. Previously reported accuracy for this method is as low as $0.25 \mathrm{~mm} .{ }^{11}$ With the hidden target test using pelvic part of the Rando phantom, we demonstrated the end-to-end testing of positioning accuracy of the complete system as a single entity. For the clinical utilization of the system, it must be remembered that precise patient positioning and accurate image fusion play a crucial role for the localization of isocenter. The ExacTrac infra-red system also add to the precision of the treatment up to 0.3 $\mathrm{mm} .{ }^{12}$ Our linear accelerator unit was tested over 6 months, the coincidence between isocenter and Exactrac imaging isocenter was found to be within $1.1 \pm 0.30 \mathrm{~mm}$. Both the $\mathrm{kV}$ and MV imaging was within $1.2 \mathrm{~mm}$ accuracy. The results obtained with these set of tests were stable, consistent and comparable with the results of the other groups. These set of quality control tests retains the phantoms and all other test tools that were used previously. We accessed the quality of images receiving from the system for uniformity, noise, contrast and spatial resolution. Using ImageJ software, the parameters were determined directly on the acquired images, providing quicker and simpler procedure. Similarly other tests for the characteristics of the $\mathrm{x}$-ray tubes were performed. These set of tests determine the $\mathrm{x}$-ray tube potential, current and timer accuracy and their reproducibility. ${ }^{13} \mathrm{With}$ these tests we provided the doses estimation for the exposures for variable potential and tube current. The inherent and applied filtration of the $\mathrm{x}$-ray units were also determined using half value layer (HVL) evaluation technique. Thus, this hybrid system was evaluated using a comprehensive quality assurance program to determine its complete performance characteristics.

\section{Conclusion}

The role of various imaging technologies is being explored across the continuum of radiotherapy treatments and a successful technology is also adopted very at a quick pace internationally. ExacTrac patient positioning system enables faster inter- as well as intra-treatment set-up for patients accurately. We performed the systematic evaluation of the Synergy Platform and ExacTrac patient positioning system for image guided radiotherapy (IGRT). Our results provide a satisfactory performance for the hybrid system and it can be considered as a single entity.

\section{Conflict of interest}

The authors declare that they have no conflicts of interest. The authors alone are responsible for the content and writing of the paper. 


\section{Acknowledgement}

Authors would like to sincerely thank Mr. Jeen SP Sathiya and Mr. Saneg Krishnankutty for their support in the preparations during the study.

\section{References}

1. Fuss M, Salter BJ, Cavanaugh SX, et al. Daily ultrasound-based image-guided targeting for radiotherapy of upper abdominal malignancies. Int J Radiat Oncol Biol Phys 2004; 59:1245-56.

2. Lattanzi J, McNeeley S, Hanlon A, et al. Ultrasound-based stereotactic guidance of precision conformal external beam radiation therapy in clinically localized prostate cancer. Urology 2000; 55: 73-8.

3. Verellen D, Soete G, Linthout N, et al. Quality assurance of a system for improved target localization and patient set-up that combines real-time infrared tracking and stereoscopic X-ray imaging. Radiother Oncol 2003; 67:129-41.

4. Wang LT, Solberg TD, Medin PM, Boone R. Infrared patient positioning for stereotactic radiosurgery of extra-cranial tumors. Comput Biol Med 2001; 31:101-11.

5. Lee SW, Jin JY, Guan H, et al. Clinical assessment and characterization of a dual tube kilovoltage X-ray localization system in the radiotherapy treatment room. J Appl Clin Med Phys 2008; 9: 2318.
6. Antonuk LE. Electronic portal imaging devices: a review and historical perspective of contemporary technologies and research. Phys Med Biol 2002; 47:R31-65.

7. Parent L, Fielding AL, Dance DR, et al. Amorphous silicon EPID calibration for dosimetric applications: comparison of a method based on Monte Carlo prediction of response with existing techniques. Phys Med Biol 2007; 52: 3351-68.

8. AAPM. Basic quality control in diagnostic radiology. AAPM Report No. 4, 1981.

9. AERB. Acceptance and performance test report for diagnostic X-ray unit. AERB/RSD/MDX/ATR/2009.

10. Lutz W, Winston KR, Maleki N. A system for stereotactic radiosurgery with a linear accelerator. Int J Radiat Oncol Biol Phys 1988; 14: 373-81.

11. Grimm J, Grimm SL, Das IJ, et al. A quality assurance method with sub-millimeter accuracy for stereotactic linear accelerators. J Appl Clin Med Phys 2011; 12: 182-98.

12. Wang LT, Solberg TD, Medin PM, et al. Infrared patient positioning for stereotactic radiosurgery of extracranial tumors. Comput Biol Med 2001; 3:101-11.

13. Sprawls P. Physical Principles of Medical Imaging, Aspen, ISBN 0-87189-644-3, Maryland, USA (1987). 